INRA, Prod. Anim., 2005, 18 (2), 87-99

\title{
Le comportement social des bovins et ses conséquences en élevage
}

\author{
M.-F. BOUISSOU ${ }^{1}$, A. BOISSY ${ }^{2}$ \\ 1 INRA, Physiologie de la Reproduction et des Comportements, UMR85, F-37380 Nouzilly \\ 2 INRA, Unité de Recherches sur les Herbivores, F-63122 Saint-Genès Champanelle \\ Courriel : boissy@clermont.inra.fr
}

\begin{abstract}
L'organisation sociale des bovins repose sur deux types de relations : celles de dominance, qui participent à la résolution à moindre coût des conflits en canalisant l'agressivité, et celles d'affinité qui assurent la cohésion du groupe en atténuant les éventuelles tensions. Certaines conduites d'élevage peuvent perturber l'organisation sociale et être facteurs de stress. Des aménagements sont proposés pour assurer la stabilité des relations de dominance et privilégier les relations d'affinité. Ils devraient faciliter l'intégration de l'animal à son groupe d'élevage et concilier ainsi bien-être et production.
\end{abstract}

Au cours des dernières décennies, l'étude du comportement des animaux domestiques s’est développée pour mieux répondre aux impératifs de production et à l'évolution des systèmes d'élevage. Cette évolution s'est faite en partie dans le sens d'une intensification qui a eu pour conséquence d'imposer aux animaux de multiples contraintes tant physiques que sociales. Leurs conditions de vie deviennent de plus en plus éloignées des conditions naturelles. Les bovins se sont adaptés à différents environnements correspondant à différents systèmes de gestion qui peuvent être considérés comme des «niches écologiques» dans lesquelles différents types d'animaux ont évolués (par exemple les races laitières ou à viande). L'évolution a été considérablement accélérée grâce à l'efficacité de la sélection des sujets les mieux adaptés par les éleveurs, et au nombre de jeunes qui atteignent la maturité sexuelle. L'animal domestique est caractérisé par une grande souplesse d'adaptation, mais ses capacités ont sans nul doute des limites. Les techniques modernes d'élevage, qui tiennent compte des impératifs économiques immédiats plutôt que des possibilités réelles des animaux, risquent de dépasser les capacités d'adaptation de l'animal faute d'une connaissance suffisante de ses exigences éthologiques.

Dans le but de rentabiliser les productions animales, la réduction de la main-d'œuvre, la constitution d'unités de taille importante d'animaux de même âge, et le recours à la mécanisation des différentes interventions humaines (distribution alimentaire, traite, nettoyage...) se sont généralisés. Il devient alors de plus en plus difficile de s'attacher aux cas particuliers en raison de la réduction très importante du contact entre l'éleveur et ses animaux : l'individu s'efface devant le groupe. Or, de telles évolutions dans les conduites d'élevage entraînent l'apparition de problèmes nouveaux dus en particulier aux tensions sociales qui s'exercent entre les animaux conduits en confinement. La connaissance des phénomènes responsables de la stabilité des relations et de la tolérance entre animaux devient alors très importante à développer. A côté des systèmes de conduites intensives, l'utilisation des herbivores sur des zones agricoles à faible productivité suscite un regain d'intérêt en raison de la nécessité sociétale du maintien d'une activité agricole dans des zones défavorisées et de la gestion de la biodiversité. Le développement de l’élevage en conditions extensives implique de réduire le gardiennage et les investissements ; le rôle de l'animal et son adaptation au milieu sont alors déterminants pour lui assurer une relative autonomie. La connaissance du comportement, en particulier la connaissance des phénomènes régissant la cohésion du groupe social et la stratégie d'utilisation de l'espace, est dans ce cas très importante.
Les bovins domestiques, comme la plupart des ruminants, appartiennent à une espèce sociale et tolèrent en particulier d'être entretenus en groupe à des densités relativement élevées. A l'opposé la conduite en isolement entraîne des troubles qui révèlent l'importance des contacts sociaux pour ces animaux. Cependant, les troupeaux constitués par l'élevage moderne diffèrent beaucoup de ceux que l'on peut rencontrer chez les Bovinés sauvages correspondants ou apparentés. Ces différences résident en particulier dans le «milieu» où évoluent les animaux (surface souvent réduite, localisation dans l'espace et dans le temps des sources de nourriture...), et dans la structure même du groupe (séparation plus ou moins précoce du jeune animal de sa mère et des autres adultes, ségrégation des sexes, constitution de lots homogènes et remaniements fréquents...). L’animal doit donc s’adapter à un milieu de plus en plus éloigné du milieu naturel de l'espèce. Il en résulte dans certains cas un inconfort physique et/ou psychologique, des troubles plus ou moins graves des comportements sexuel et maternel, et un accroissement des comportements d'agression. Une étude approfondie du groupe social, en particulier de sa structure, des mécanismes intimes des relations interindividuelles, et de son rôle d'interface entre l'individu et son environnement non social, est donc essentielle pour mettre à profit les potentialités sociales des bovins domestiques et proposer des conduites d'éle- 
vage qui non seulement tiennent compte des impératifs économiques, mais également respectent le bien-être animal.

Cet article fait le point des connaissances concernant les caractéristiques des relations sociales chez les bovins. Nous rappellerons tout d'abord les fondements de leur comportement social et de celui de leurs proches parents dans les conditions plus ou moins naturelles. Dans une seconde partie, nous décrirons les caractéristiques des relations sociales des bovins dans les conditions de l’élevage moderne. Dans une troisième partie, nous envisagerons les conséquences de ces relations en terme de gestion et de bien-être des bovins en élevage.

\section{1 / Le comportement social des Bovinés}

\section{1 / La structure sociale}

Avant d'aborder l'étude du comportement des bovins domestiques tels qu'ils sont élevés dans les conditions commerciales, il est utile de donner un bref aperçu des structures sociales de leurs plus proches parents et des bovins domestiques retournés à l'état sauvage, puisque malheureusement le genre Bos n'existe plus à l'état sauvage. L'ancêtre des Bovins, l'auroch (Bos taurus primigenius, Bojanus), est en effet éteint depuis le XVIIe siècle.

Les bovins appartiennent à l'ordre des Ongulés et à la famille des Bovidés. Cette famille comporte 14 sousfamilles, dont celle des Bovinés qui regroupe les bovins, les bisons d'Amérique et d'Europe, les buffles asiatiques et africains, le yack et le bœuf musqué. Au sein de la sousfamille des Bovinés, le genre Bos ne comporte de nos jours que les espèces domestiques Bos taurus et Bos indicus (zébu), dont la domestication remonte à 6000 ans avant J.C.

Bien qu'il y ait peu de populations de bovins retournées à l'état sauvage, leur observation permet d'appréhender ce que fut la structure sociale de l'ancêtre des bovins domestiques. Parmi elles, citons les bovins introduits par des explorateurs au XIX ${ }^{\mathrm{e}}$ siècle dans 1'lle d'Amsterdam au Sud-Est de Madagascar (Daycard 1990), le bétail Maremm en Italie (Lucifero et al 1977), une population d'environ 160 individus au sud de l'Espagne (Lazo 1994) et une autre dans les îles Orkney
(Hall et Moore 1986). Un cas particulier est celui du bétail blanc de Chillingham, qui vit pratiquement sans intervention humaine dans un parc clos depuis plus de 700 ans (Hall 1986). Enfin certains animaux sont entretenus en semi-liberté avec des interventions minimales, comme les bovins de Camargue (Schloeth 1961).

Les Bovinés ne sont pas territoriaux. Les caractéristiques principales de leur structure sociale sont l'intégration possible des mâles et des femelles dans des troupeaux mixtes, la précocité des jeunes et des distances sociales faibles. Trois types de groupes sont généralement observés (Daycard 1990) : 1) des groupes de femelles de tous âges accompagnées de leurs jeunes et de quelques mâles sub-adultes, 2) des petits groupes de mâles adultes et subadultes sachant qu'une forte proportion des mâles adultes est solitaire, et 3) des groupes mixtes, surtout pendant la période de reproduction (figure 1). La cohésion des groupes de mâles est moindre que celle des groupes de femelles. Mâles et femelles occupent parfois des domaines vitaux différents, qui sont appris précocement par les jeunes. Dans certaines circonstances, la réunion de plusieurs groupes conduit à la formation de troupeaux de taille très importante pouvant aller jusqu'à plusieurs centaines d'individus, voire plusieurs milliers, comme c'est le cas chez le bison (Lott et Minta 1983). Une des caractéristiques des Bovinés est l'existence d'une hiérarchie entre les mâles, les femelles ayant leur propre système hiérarchique ; mais en général les mâles adultes dominent les femelles. Enfin, lorsqu'il y a des mâles adultes au sein du troupeau, ils ne sont pas forcément les dominants, ni les leaders, et ils n’ont pas non plus d'action sur la cohésion du groupe.

\section{2 / La communication}

Le comportement d'un individu s'organise entre autres grâce aux informations qui lui parviennent du milieu dans lequel il vit. Certaines de ces informations proviennent des autres animaux de la même espèce et le renseignent sur leur identité, leur «humeur», leurs «intentions»; lui-même émet des signaux. Ainsi se crée la communication indispensable à toute vie sociale à partir du moment où les partenaires répondent à ces signaux.

Chez les Bovinés, les signaux visuels sont importants comme véhicules de l'information dans les relations sociales; il s'agit sans doute d'une adaptation à la vie des steppes, milieu naturel de nombreuses espèces, et au statut de proie, alors que chez celles vivant en milieu forestier, la vue joue un rôle secondaire. L'acuité visuelle ne serait que d'environ 1/20 de celle d'un humain (Entsu et al 1992). Par contre, et contrairement à une croyance répandue, la vision des couleurs a été clairement démontrée par des expériences de conditionnement opérant (Riol et al 1989). La musculature faciale étant relativement pauvre, le jeu des mimiques est restreint par rapport à ce qu'il peut être chez d'autres Ongulés comme les chevaux par exemple. Par contre, la mobilité de la tête par rapport

Figure 1. Représentation schématique de l'évolution de la structure sociale pendant la période de reproduction chez les Ongulés non territoriaux et ne formant pas de harems permanents.

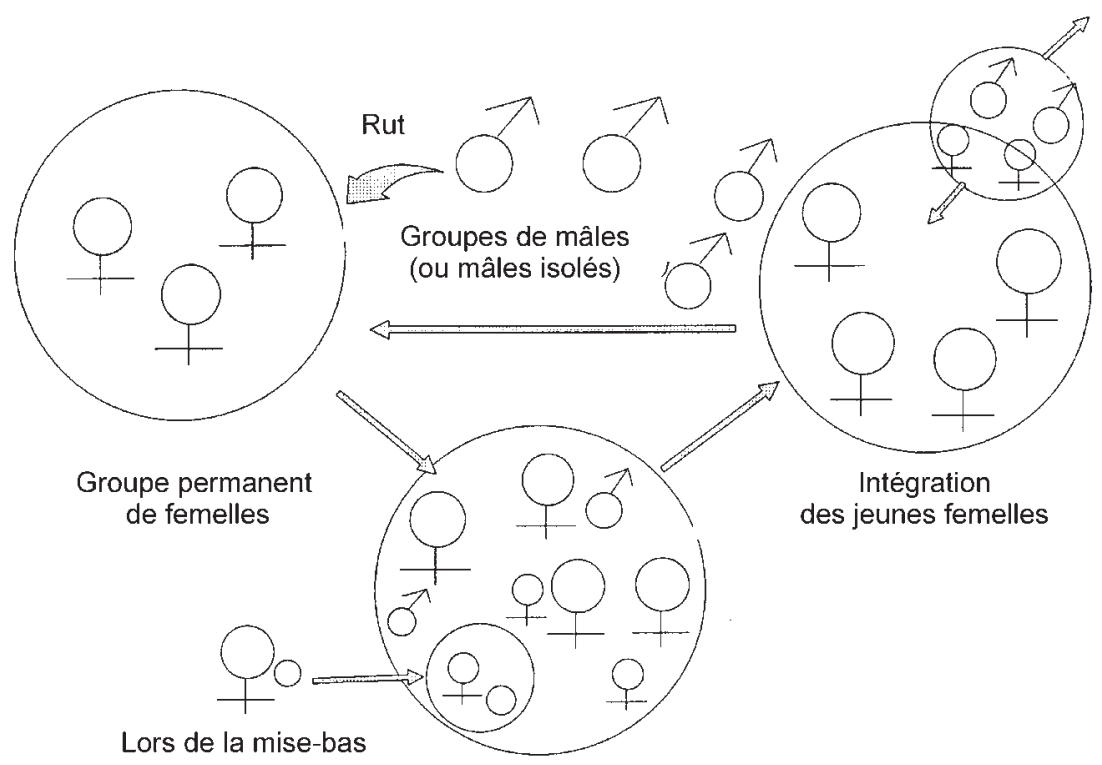


au corps permet des parades où la position de celle-ci joue un rôle essentiel (Schloeth 1958).

Différents types de vocalisations ont été décrits grâce à l’étude de sonogrammes. Contrairement aux mâles qui émettent des meuglements de menace, les vocalisations accompagnent assez rarement l'agression chez les bovins femelles. Les vocalisations sont le plus souvent provoquées chez elles par une situation de frustration, d'excitation, ou l'appel d'un autre animal (Kiley 1972).

Le nombre considérable de glandes odoriférantes présentes chez les bovins suggère l'importance de l'olfaction dans leur vie sociale bien que leurs capacités olfactives n'aient fait l'objet que de rares études. Pourtant, les bovins comme les caprins, les ovins et les porcins sont capables de faire la distinction entre deux individus en ne se basant que sur l'odorat (Baldwin 1977). Par contre, ni l'établissement ni le maintien des relations sociales n'est affecté chez des vaches privées du sens de l'odorat par l'ablation des bulbes olfactifs (Bouissou 1985). Le fait d'asperger des vaches avec une substance odorante (huile anisée) réduit la fréquence des interactions agressives et la production laitière consécutive à un regroupement (Cummins et Myers 1991). En outre les animaux peuvent communiquer leur état psychologique,

en particulier leur peur, au moyen de phéromones. Des génisses ont un comportement alimentaire perturbé en présence de congénères stressés (Boissy et al 1998) et leur latence pour s’approcher d'un distributeur de nourriture est augmentée en présence d'urine de congénères stressés (figure 2).

Il est cependant exceptionnel qu'un signal isolé soit seul en cause. Très souvent plusieurs organes des sens sont stimulés en même temps : par exemple une perception olfactive accompagne en général une perception visuelle. On constate une action cumulative des différents signaux et la possibilité de substitution entre eux. Un comportement adapté peut donc, dans la majorité des cas, s’organiser à partir d'un nombre restreint d'informations, ou même en l'absence des informations d'un canal sensoriel.

\section{3 / Les actes et postures utilisés dans les relations sociales}

Ils se divisent en deux grandes catégories : d'une part, les actes agressifs (dits offensifs) et ceux qui répondent à l'agression (soumission, retrait, fuite), qui sont regroupés sous le terme $d$ 'actes agonistiques ; d'autre part les actes dits non agonistiques parmi lesquels on peut distinguer ceux faisant partie du répertoire sexuel et tous les autres, dont les comportements d'affinité (Bouissou 1985).

Figure 2. Transmission de l'état de stress entre bovins (Boissy A., Terlouw C., Le Neindre P. 1998).

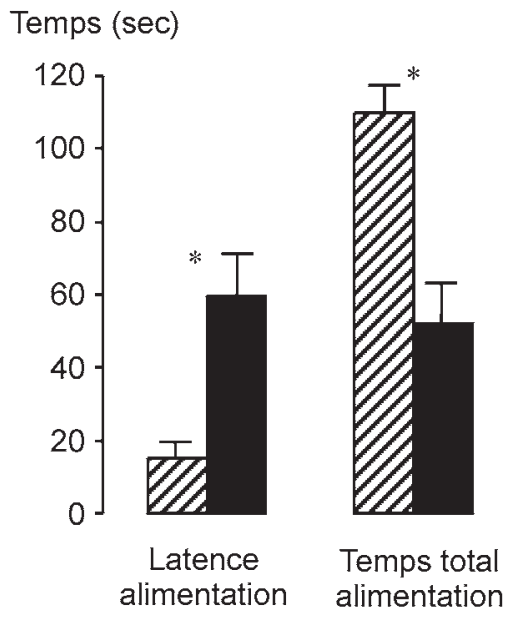

a

$$
\text { Temps (sec) }
$$

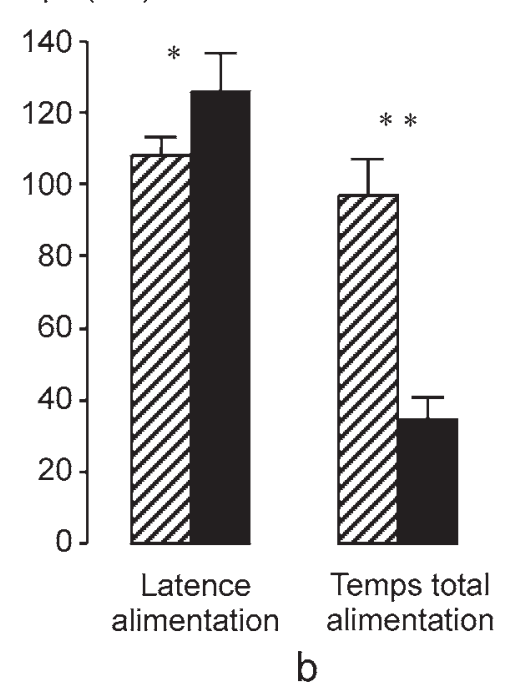

a) Réactions de génisses placées dans un environnement non familier dans lequel elles peuvent s'alimenter, en présence de congénères stressés (surface noircie) ou non stressés (surface hachurée).

b) Réactions de génisses placées dans un environnement non familier en présence d'urine de congénères stressés ou non stressés, l'urine étant placée sous la nourriture.
Les actes agressifs incluent les combats, les coups, et les menaces (figure 3a, b, c, d). Les coups sont donnés avec la tête, et sont en général dirigés vers le flanc et l'arrière-train de l'adversaire ; ils sont plus ou moins violents et la victime peut, dans certains cas, être blessée surtout si l'attaquant possède des cornes et que la fuite est impossible. Les menaces sont des actes offensifs n'aboutissant pas obligatoirement à un contact physique. C'est selon les cas un simple mouvement de tête de côté, ou des attitudes agressives plus sophistiquées et qui entraînent généralement le retrait de celui à qui elles sont adressées. Une attitude très particulière dite «menace en position latérale» (figure 3g) est commune à tous les bovidés : l'animal menaçant tourne lentement sur luimême et se présente de profil à son adversaire, cette menace se termine soit par le départ de ce dernier ou de l'animal menaçant lui-même, soit par une brusque volte-face et une attaque. C'est une attitude ambivalente qui exprime à la fois une tendance à la fuite et à l'attaque. Parfois un animal riposte à la suite d'un coup reçu, ou répond à une menace par une autre menace, et dans ce cas un combat peut s'engager. Les combats véritables restent cependant rares et sont limités aux premières rencontres entre animaux étrangers. La durée des combats est extrêmement variable ; elle peut aller de quelques secondes à plus d'une heure. Dans la majorité des cas cependant elle est inférieure à une minute. Enfin, les combats entre vaches donnent lieu à des manifestations folkloriques traditionnelles dans certaines régions des Alpes (en particulier le Valais Suisse).

A l'opposé des actes offensifs, on trouve les actes défensifs qui, chez les bovins, consistent exclusivement en des mouvements orientés à l'opposé de l'agresseur. Il peut s’agir par ordre d'intensité croissante d'évitements et de fuites. Un évitement consiste en un simple détournement de la tête ou d'un abaissement de la tête, l'angle encolure-chanfrein ouvert par rapport à la position normale (et donc à l'inverse de la position de menace); ce comportement étant parfois accompagné d'un recul (figure 3d). Dans le cas de la fuite, l'activité locomotrice est plus importante (figure 3c, e, f). Lorsque les relations de dominance-subordination sont bien établies au sein d'un groupe, ces fuites et évitements spontanés représen- 
Figure 3. Interactions agonistiques.

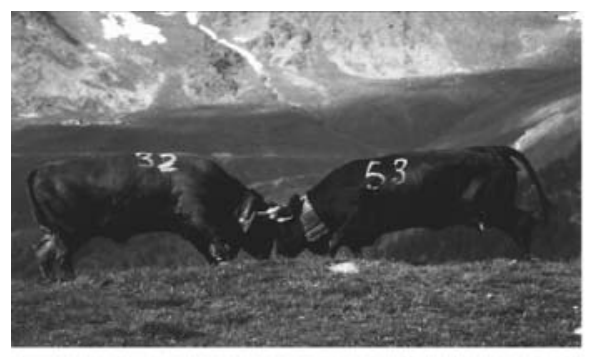

a

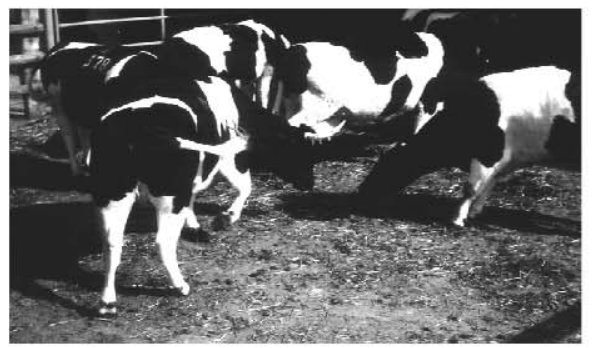

d

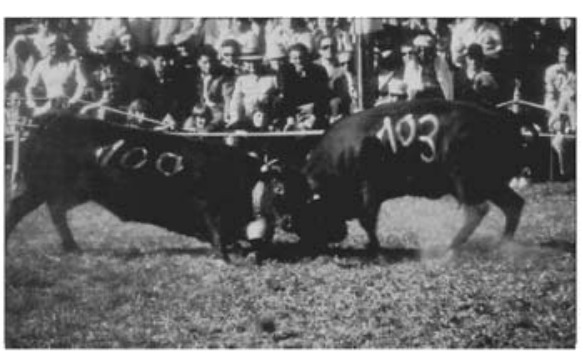

b

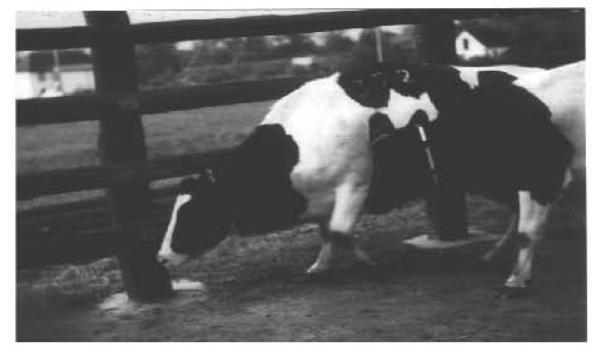

e

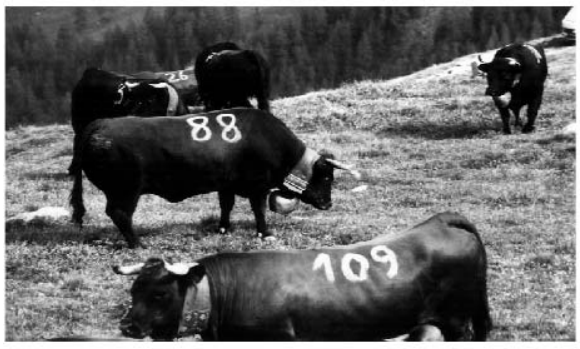

g

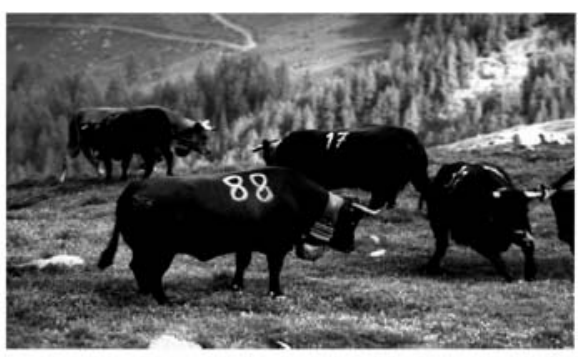

C

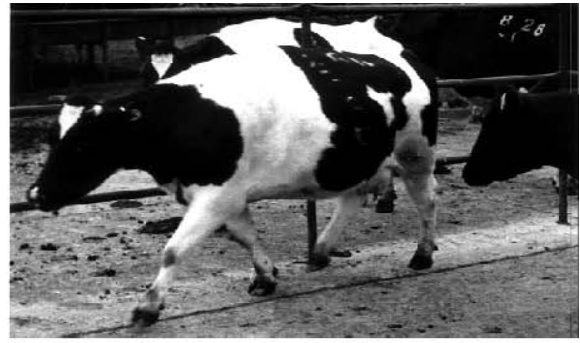

f

a) Lutte entre vaches de la race d'Hérens à l'alpage ; b) Lutte entre vaches de la race d'Hérens lors des spectacles organisés ; c) menace (animal $\mathrm{n}^{\circ} 88$ ) et fuite de son adversaire ; d) Menace de l'animal de gauche et attitude de soumission de celui de droite ; e, f) Fuites ; g) menace latérale (animal $\left.n^{\circ} 88\right)$.

tent la part la plus importante des actes agonistiques.

Parmi les actes non agonistiques on trouve chez la femelle, un comportement de monte analogue à celui du mâle, et qui fait partie du répertoire comportemental normal de la vache quel que soit son état physiologique. La réceptivité sexuelle, lors de l'œstrus, se traduit par une immobilisation en réponse aux tentatives de monte de ses congénères mâles ou femelles.

Enfin, il existe d'autres actes non agonistiques tels que les flairages, les léchages, les frottements, les appuis de la tête contre un autre animal, les «jeux de tête»... Ces comportements de «toilettage» mutuel (allogrooming) qui s'expriment entre autres dans les relations d'affinité, remplissent une fonction sociale en réduisant les tensions et en resserrant les liens sociaux. Selon les auteurs, le léchage est le fait du dominé ou du dominant d'une paire. Il existe des partenaires préférés pour cette activité.

La proportion d'interactions représentée par les interactions agonistiques diminue lorsque le groupe est constitué depuis longtemps et, dans ce cas, ce sont surtout les fuites et les évitements spontanés qui s'expriment le plus. Cependant, le mode d'entretien intervient également : lorsque les vaches sont en stabulation, les interactions agonistiques constituent la majorité des interactions échangées, voire la quasi-totalité ; au contraire, les mêmes animaux placés au pâturage échangent proportionnellement plus d'actes non agonistiques que d'actes agressifs.

\section{2 / Les relations sociales des bovins}

\section{1 / Les relations de dominance- subordination}

En condition naturelle, au sein des groupes permanents, des conflits peuvent surgir pour l'alimentation, la reproduction, l'occupation d'un site favorable... Des mécanismes régulateurs ont évolué pour limiter les conduites agressives ou les orienter vers des formes bénignes moins préjudiciables aux individus et à l'espèce. C'est ainsi que se sont développés les phénomènes de dominance qui assurent la priorité d'accès de certains individus à des ressources limitées, sans qu'il soit besoin de recourir à chaque occasion à une épreuve de force : la dominance d'un animal est reconnue par le ou les subordonnés du groupe. Le raffinement 
des moyens de communication permet soit au dominant d'affirmer sa position de manière non violente, soit au subordonné de montrer son acceptation avant même toute démonstration agressive de la part du dominant.

Les relations de dominancesubordination ont fait l'objet de très nombreuses études chez les bovins. Un animal dominant est défini comme celui qui peut attaquer sans être agressé, et qui jouit de priorité lorsqu'il y a compétition pour une ressource limitée. La notion de dominance est cependant à distinguer de celle d'agressivité (l'animal dominant n'est pas forcément un animal agressif) et du leadership (l'animal dominant n'est pas forcément celui qui conduit le groupe). Une des caractéristiques importantes de la dominance chez les bovins, et surtout chez les vaches, est qu'elle est de type «absolu» : seul le dominant de chaque dyade agresse alors que le subordonné fuit (Bouissou 1985), à l'inverse de se qui se passe dans d'autres espèces, comme le porc par exemple chez lequel les deux peuvent attaquer bien que de manière très inégale. Une patiente observation de toutes les interactions agonistiques survenant dans le groupe non perturbé est donc la meilleure méthode pour connaître les relations entre ses membres. Néanmoins, cette méthode peut s'avérer longue et fastidieuse lorsque le groupe est de taille importante, et/ou constitué depuis longtemps car les agressions y sont alors rares. D’autres méthodes ont été développées : elles reposent sur une compétition, le plus souvent alimentaire, à laquelle sont successivement soumises

Figure 4. Epreuve de compétition alimentaire.

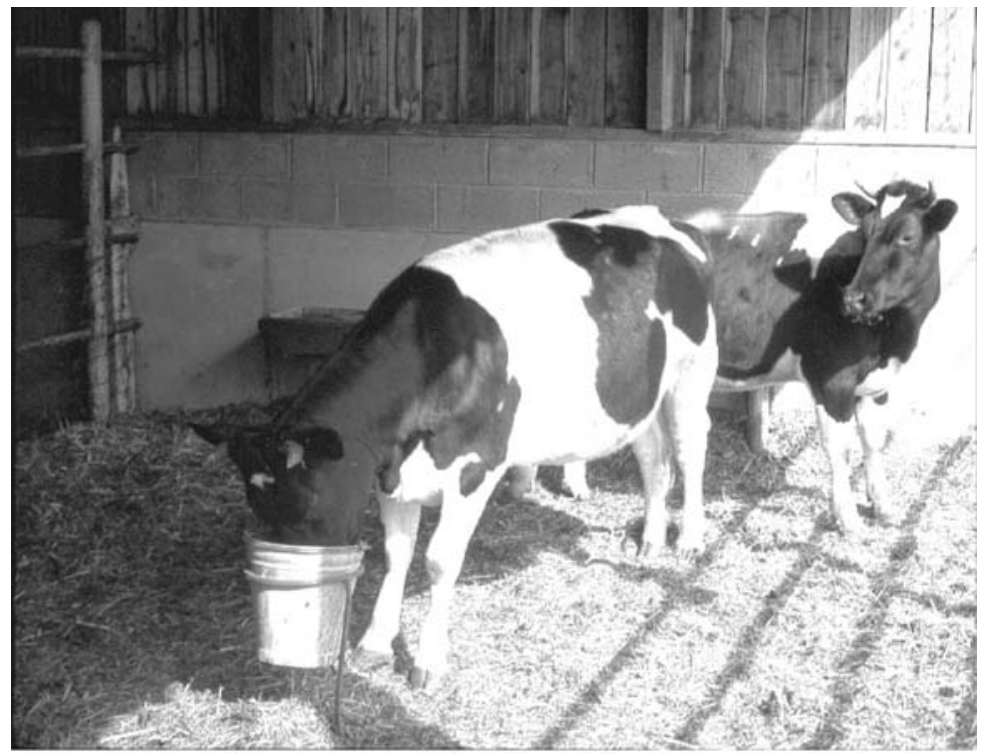

Seul le dominant a accès à l'aliment contenu dans le seau. Le plus souvent le dominé ne tente même pas de s'approcher.

Figure 5. Exemples de structures hiérarchiques observées dans des groupes de bovins femelles.
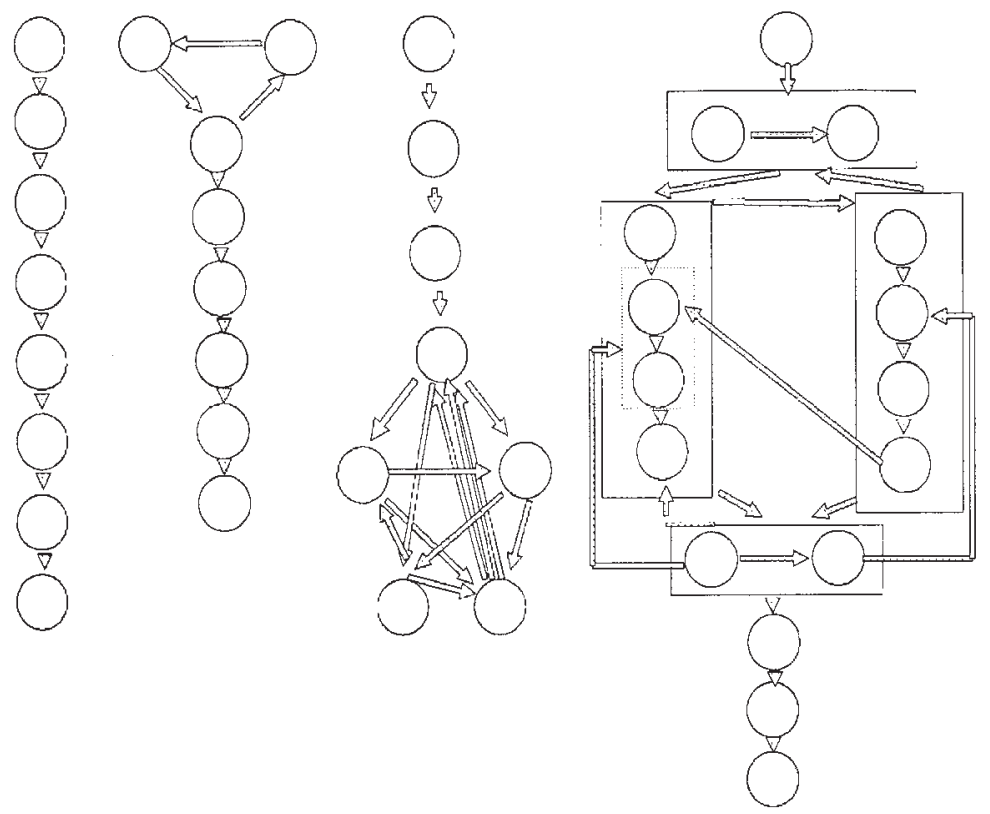

Chaque animal est représenté par un cercle. Le sens des flèches indique le sens de la dominance ; si les relations entre deux animaux ne sont pas précisées, par convention un animal domine ceux qui sont situés en dessous sur le schéma. toutes les paires possibles du groupe (figure 4).

La structure du groupe est la résultante de l'ensemble des relations de dominance-subordination dyadiques entre les individus qui le constituent. Elle peut revêtir différentes formes depuis les hiérarchies strictement linéaires où un individu domine tous les autres, et un autre est dominé par tous, jusqu'à des structures très complexes (figure 5).

Les relations de dominancesubordination s'établissent soit au cours du développement du jeune animal, soit au contraire lorsque des animaux adultes étrangers les uns aux autres sont réunis pour la première fois. Ce dernier cas, rare dans la nature, est au contraire très fréquent pour les animaux domestiques. Dans les conditions naturelles comme par exemple dans les troupeaux en Camargue, le jeune animal s'intègre dans une structure sociale préexistante (Schloeth 1961). Lorsque les animaux sont très jeunes les interactions agonistiques tendent à être bi-directionnelles et, de plus, les coups n'entraînent pas toujours la fuite de l'animal qui les reçoit. Les relations de dominancesubordination entre les jeunes bovins s'établissent à un âge plus ou moins précoce, en fonction de l'expérience de l'animal et du contexte social (Bouissou 1985). Ainsi, les veaux élevés par leur mère, et en présence d'autres adultes, établissent des relations de dominancesubordination plus précocement que des veaux élevés artificiellement en groupe de jeunes, car ils apprennent plus rapidement à utiliser les moyens de communication. Chez les femelles, l'apparition des relations de dominancesubordination correspond dans la majorité des cas à l'apparition des premières chaleurs (figure 6). Des relations nettes et stables s'établissent beaucoup plus tardivement chez les mâles.

Lorsque des animaux adultes étrangers les uns aux autres sont regroupés, le fait le plus frappant est la rapidité d'établissement des relations de domi- 
Figure 6. Etablissement des relations de dominance au cours de l'ontogenèse de génisses (Bouissou M.-F. 1977).

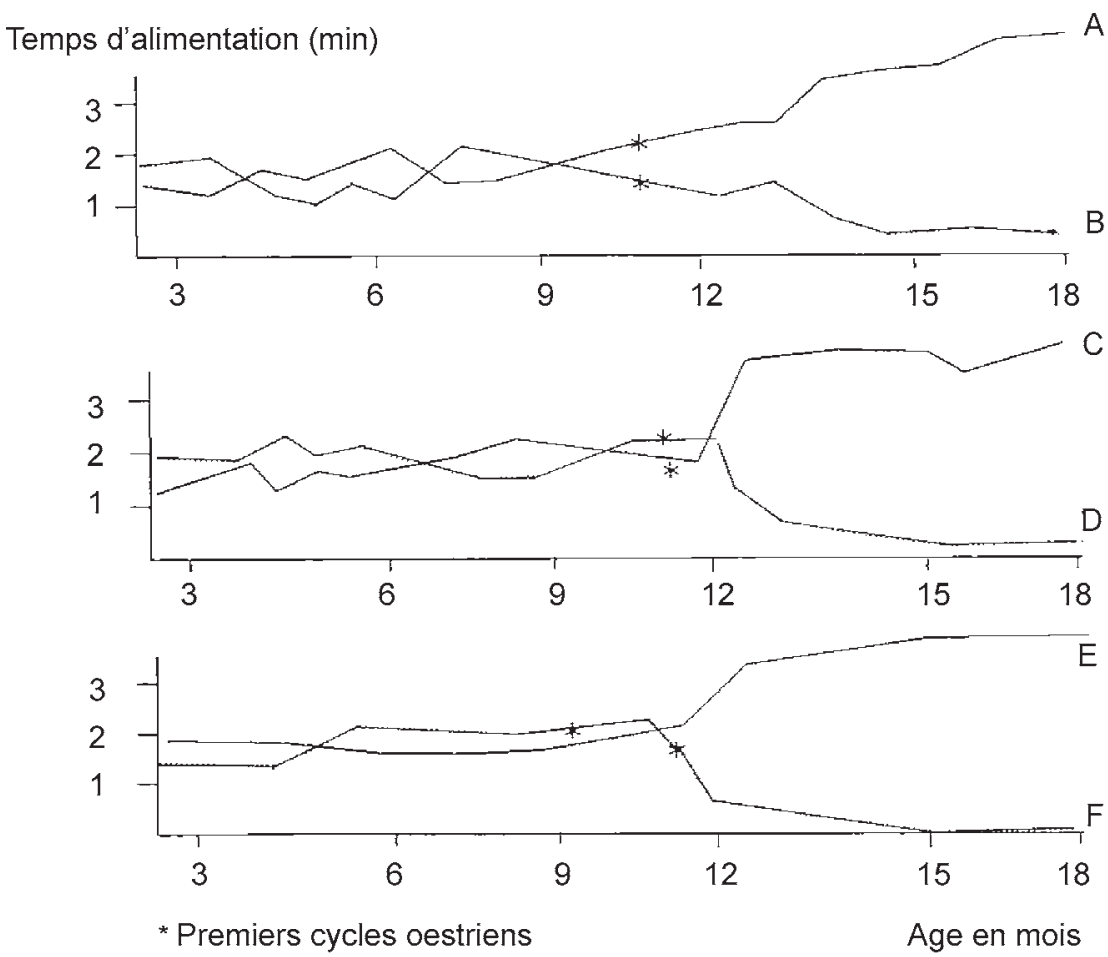

Evolution des temps d'alimentation de génisses de troupeau laitier, opposées par paires dans une situation de compétition alimentaire depuis le sevrage jusqu'à 18 mois. Au début les animaux mangent pendant un temps sensiblement égal, puis la dominance d'un animal sur l'autre s'affirme : cette période correspond dans $85 \%$ des cas à l'apparition des premières chaleurs.

nance-subordination : $60 \%$ au cours des 10 premières minutes dans de petits groupes, et la totalité au cours de la première heure (figure 7). Les combats n’interviennent que dans un tiers des cas seulement, et des relations peuvent même s'établir en l'absence de tout contact physique (Bouissou 1985). L'expérience sociale acquise par les animaux joue un rôle très important ; elle rend l'établissement des relations de dominance-subordination de plus en plus rapide et de moins en moins violent (figure 8).

Ces relations entre bovins femelles adultes sont extrêmement stables et peuvent persister pendant plusieurs années, ni l'œstrus ni la gestation ne les modifie (Bouissou 1985). Par contre, elles sont moins stables entre jeunes et entre mâles adultes. Ni la vue ni l'odorat ne sont nécessaires au maintien des relations (Mansard et Bouissou 1980). Cependant, le contact physique, bien que non nécessaire dans les conditions normales, doit rester possible (Bouissou 1985). Sa suppression totale entraîne la disparition de la réaction d'évitement du dominé à la suite des menaces du dominant, et il n'est plus possible de mettre en évidence les relations de dominance-subordination lors d’une situation de compétition alimentaire. Il existe donc chez les bovins un «sentiment de protection» vraisemblablement acquis au cours des expériences similaires qu'un animal peut faire au cours de sa vie en stabulation ou au pâturage. Lorsque le contact physique n’est pas totalement supprimé mais seulement réduit, la protection doit se situer au niveau de la tête. L'importance pratique de ces phénomènes est évidente pour la conception et l'aménagement de bâtiments, de dispositifs d'alimentation...

La connaissance des facteurs qui déterminent ou influencent la position sociale d'un individu est au centre de l'analyse du fonctionnement du groupe. Elle peut également permettre dans les conditions expérimentales ou dans la pratique de l'élevage des bovins domestiques, d'intervenir pour modifier ou atténuer les conséquences négatives éventuelles du rang hiérarchique. Selon la majorité des auteurs, l’âge semble important dans l'acquisition du rang social ; cependant il est souvent indissociable de l'ancienneté dans le groupe et de l'expérience (Cummins et Myers 1991, Kabuga 1992). De nombreux facteurs physiques (poids corporel, hauteur au garrot, présence ou absence de cornes...) ont été également évoqués pour rendre compte d'un rang social élevé (Kabuga 1992). Une corrélation positive entre rang social et poids ou taille a parfois été trouvée. Cependant la différence de poids peut être la conséquence et non la cause du statut social. Bien que la présence de cornes confère un avantage certain à l'animal au moment de l'établissement de la relation de dominance-subordination, l’écornage ultérieur n'a pratiquement pas d'effet contrairement à une croyance répandue. L'expérience précoce, incluant les conditions d'élevage, peut influencer la position sociale à
Figure 7. Dynamique de l'établissement des relations de dominance dans des groupes de 4 génisses étrangères les unes aux autres : effet de l'expérience sociale (Bouissou M.-F. 1975).

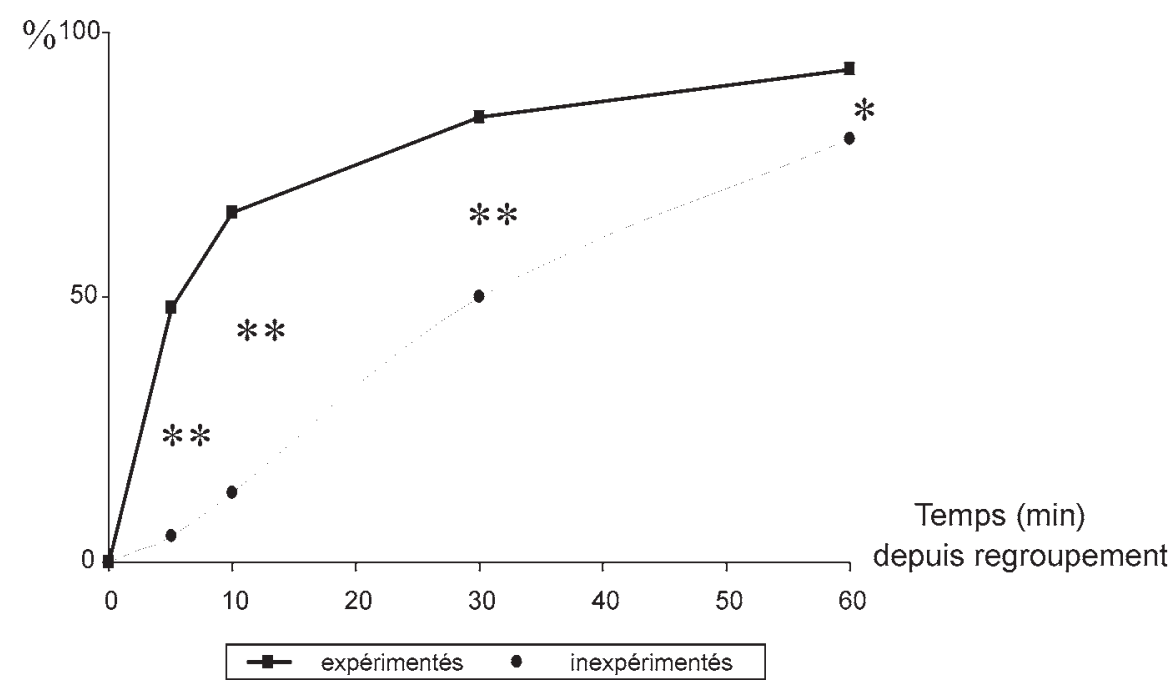

Etablissement des relations de dominance-soumission chez les bovins domestiques. III. Rôle de l'expérience sociale. Zeitschrift für Tierpsychologie 38, 419-435). 
Figure 8. Mode d'établissement des relations de dominance en fonction de l'expérience sociale (Bouissou M.-F.1975).

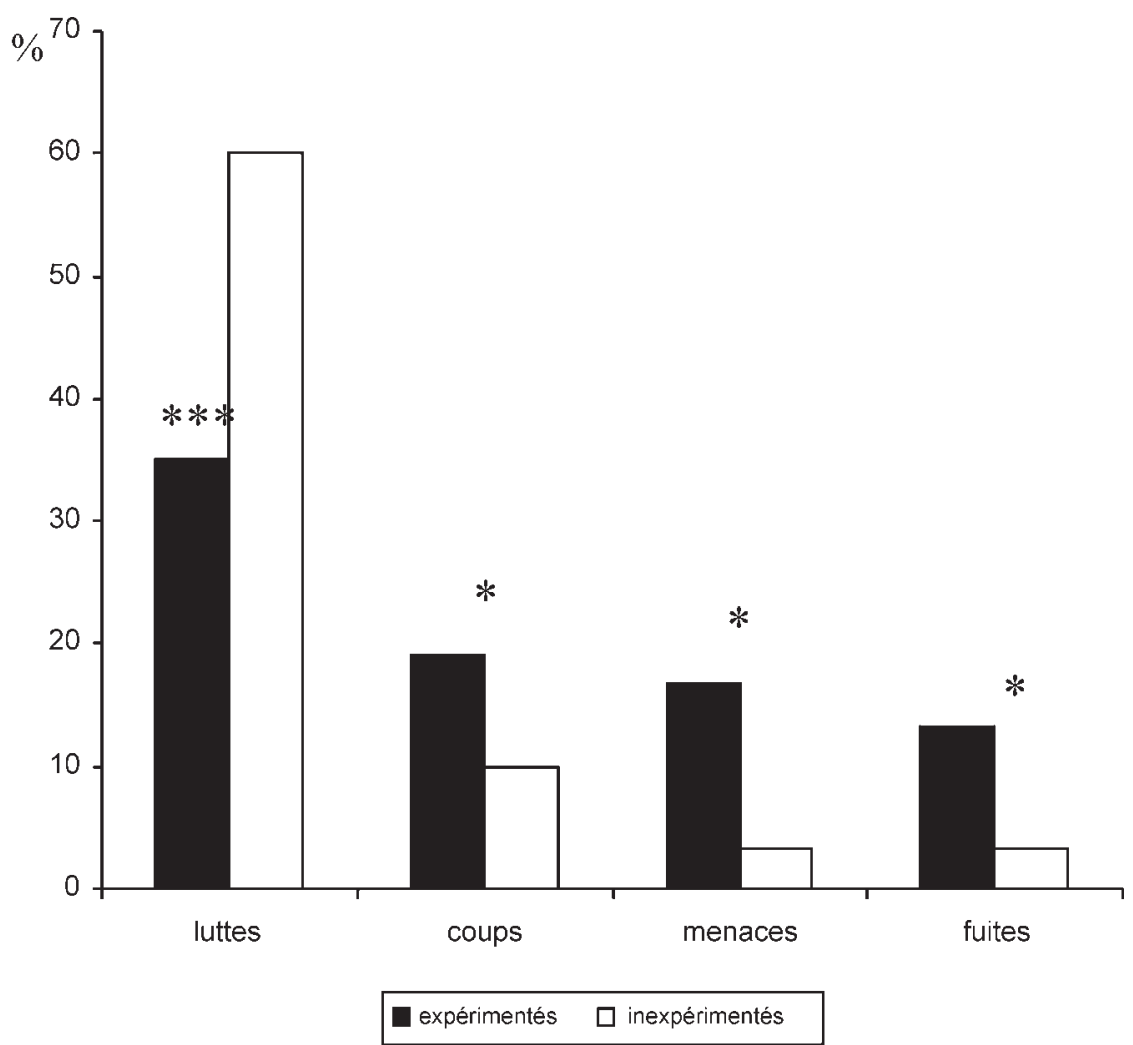

Proportion des relations établies à la suite de chacune des interactions (luttes, coups, menaces, fuites) dans des groupes de 4 génisses étrangères les unes aux autres.

l’âge adulte. Cependant une privation sociale même importante (animaux élevés dans l’isolement complet durant les 6 premiers mois) n'a pas de conséquences durables sur les relations sociales et la dominance chez les bovins, à l'opposé de ce qui est connu dans d'autres espèces (Bouissou 1985). Bien qu'ayant été l'objet de peu d'études, le rang social de la mère ne semble pas influencer celui du veau qu'elle élève (Le Neindre 1984). Par ailleurs, la dominance semble peu héritable chez des génisses élevées séparément de leurs mères. Une influence génétique sur la dominance est cependant suggérée par plusieurs faits : les animaux de certaines races dominent de manière constante les animaux d'autres races (Le Neindre et Sourd 1984, Stricklin 1983) ; par ailleurs les jumeaux occupent en général un rang identique au sein des groupes, et il est possible de les échanger entre deux groupes sans en modifier la structure, ni provoquer de combats (Bouissou 1985). La position sociale des bovins peut également être influencée par le niveau circulant de stéroïdes sexuels. Des traitements précoces à base d'androgènes ou d'œstrogènes permettent d'influencer le rang social futur d'un jeune animal : $85 \%$ des génisses ayant reçu un traitement d'androgènes entre l'âge de 3 et 6 mois sont dominantes lorsqu'elles rencontrent, à 15 mois, des étrangers non traités (Bouissou 1985). Il a également été possible de modifier complètement la structure sociale au sein de groupes stables de vaches, en traitant certaines d'entre elles par des androgènes ou des oestrogènes. Les animaux traités deviennent dans tous les cas dominants par rapport au non traités, mais ne modifient pas les relations qui existent entre eux (Bouissou et Gaudioso 1982). Enfin le «tempérament», qui inclut la réactivité émotionnelle, est probablement un des facteurs les plus importants dans la détermination du rang social (Boissy et Bouissou 1994). En effet, les animaux de rang élevé fuient les animaux qui les dominent moins fréquemment que ne le font les animaux de rang inférieur, et les veaux qui deviendront dominants ne fuient pratiquement jamais avant l'apparition des relations de dominance-subordination. Dans les études impliquant un traitement par des stéroïdes sexuels, il a été clairement démontré que la plus grande aptitude à la dominance acquise par les sujets traités, responsable de l'inversion des relations de dominance- subordination, était due à une réduction de leurs réactions de peur mesurées dans des situations sociales, mais aussi non sociales. De plus, une étude portant sur des vaches de la race d'Hérens, utilisée lors des combats de vaches en particulier en Suisse, a montré que le faible nombre de réactions de peur, mesurées à l'âge de 6 mois, est le meilleur prédicteur de la dominance à l'âge adulte (Plusquellec et Bouissou 2001).

\section{2 / Les relations d'affinité}

Chez les Bovinés, la base de la structure sociale est, dans la plupart des cas, le groupe familial d'origine matriarcale: quelques femelles et leurs filles adultes, accompagnées des jeunes des deux sexes. Il a souvent été difficile, sinon impossible, de mettre en évidence une organisation hiérarchique dans de tels groupes. Cette permanence du groupe familial et la faible agressivité observée ont souvent fait penser à une absence de structure hiérarchique et suggèrent que des relations préférentielles existent entre ses membres et en assurent la cohésion.

Dans les conditions d'élevage, on observe la formation de sous-groupes au sein des troupeaux, aussi bien en stabulation qu'au pâturage, ainsi que des associations préférentielles entre certains animaux (Reinhardt 1981). Ces préférences peuvent être réciproques et sont très stables dans le temps. Des relations «d'affinité» existent donc entre certains animaux et se traduisent à la fois par une fréquence élevée de contacts non agressifs (par exemple toilettage), une faible fréquence d'interactions agressives et une grande proximité spatiale. Elles se traduisent également, bien que plus rarement, par des déplacements en commun et une synchronisation des activités.

L’origine de ces relations préférentielles est à rechercher dans un âge voisin et dans le fait que les animaux soient arrivés en même temps dans le groupe ou proviennent du même élevage. L’origine des relations préférentielles est également liée à la parenté. C’est ainsi que chez le zébu, la mère préfère son fils ou sa fille, même devenus adultes et ayant à leur tour des descendants, à tout autre juvénile ou adulte. Inversement les animaux préfèrent leur mère aux autres adultes. Tout ceci suggère qu'un contact prolongé à un moment quelconque de l'existence, mais généralement dans le jeune âge, peut conduire à des relations préférentielles ultérieures. Des études ont en effet montré que l'élevage en commun 
depuis la naissance a des conséquences durables sur le comportement social des animaux parvenus à l'âge adulte. Lorsque des génisses non apparentées, ayant été élevées ensemble depuis la naissance, sont regroupées avec des étrangères, elles échangent avec ces dernières un nombre d'interactions agonistiques nettement supérieur à celui qu'elles échangent entre elles (Bouissou et Hövels 1976a, b). Par contre, le nombre d'interactions non agressives est inférieur. De plus, les génisses élevées ensemble restent spatialement associées aussi bien en stabulation qu'au pâturage. Ces phénomènes persistent au moins un an après la réunion des groupes d'élevage. Enfin, ces animaux font preuve d'une grande tolérance mutuelle dans une situation de compétition alimentaire. Il semble exister une période privilégiée pour la création des relations d'affinité : elle se situerait au cours des 6 premiers mois de la vie, et pourrait être limitée par l'apparition des relations de dominance-subordination (Bouissou et Andrieu 1978, figure 9).

La tolérance, qui existe entre les animaux présentant des relations d'affinité même dans une situation de compétition relativement forte, est un phénomène d'une importance considérable pour la gestion des animaux domestiques entretenus dans des conditions intensives. Il permet d'effacer en partie les conséquences défavorables de l'existence des relations de dominancesubordination pour les animaux subordonnés.

Pour conclure, le maintien des liens préférentiels établis durant le jeune âge, voire leur renforcement, permettrait aux animaux de mieux supporter les contraintes dues à la promiscuité en élevage intensif. Il permettrait de réduire les tensions sociales et d'améliorer l'homogénéité des performances. Les risques sanitaires inévitables lors de mélanges d'animaux d'origines variées seraient de plus minimisés par le maintien des groupes d'élevage. Ces phénomènes d'affinité qui assurent la cohésion du groupe social pourraient également faciliter la conduite de troupeaux de bovins non gardiennés en conditions extensives.

\section{3 / Le leadership}

Le fonctionnement du troupeau repose également sur le phénomène de leadership. Le leadership est la capacité qu'ont certains animaux d'influencer et de recruter les autres membres du groupe, qui se traduit sur le terrain par des déplacements collectifs (Meese et Ewbank 1973). Tous les animaux vivant en troupeau manifestent des mouvements ou des activités de groupe dans diverses circonstances. Le leadership est qualifié de «social» lorsqu'il concerne le contrôle de l'agression et des aspects tels que la protection des individus face à un danger, et de «spatial» lorsqu'il concerne les déplacements du groupe (Syme et Syme 1979).

Dans le leadership spatial, le leader est celui qui initie des déplacements de groupe. C'est généralement celui qui est en tête du mouvement bien qu'il ait été suggéré que les animaux responsables des mouvements puissent être à l'arrière du groupe, poussant ainsi les autres devant eux. Cependant, la capacité à pousser serait liée à la dominance alors que l’incitation des congénères à suivre dépendrait à la fois de l'attracti-

Figure 9. Effet d'un contact social précoce sur le temps d'alimentation et les agressions de génisses en situation de compétition alimentaire (Bouissou M.-F., Hövels J. 1976a).
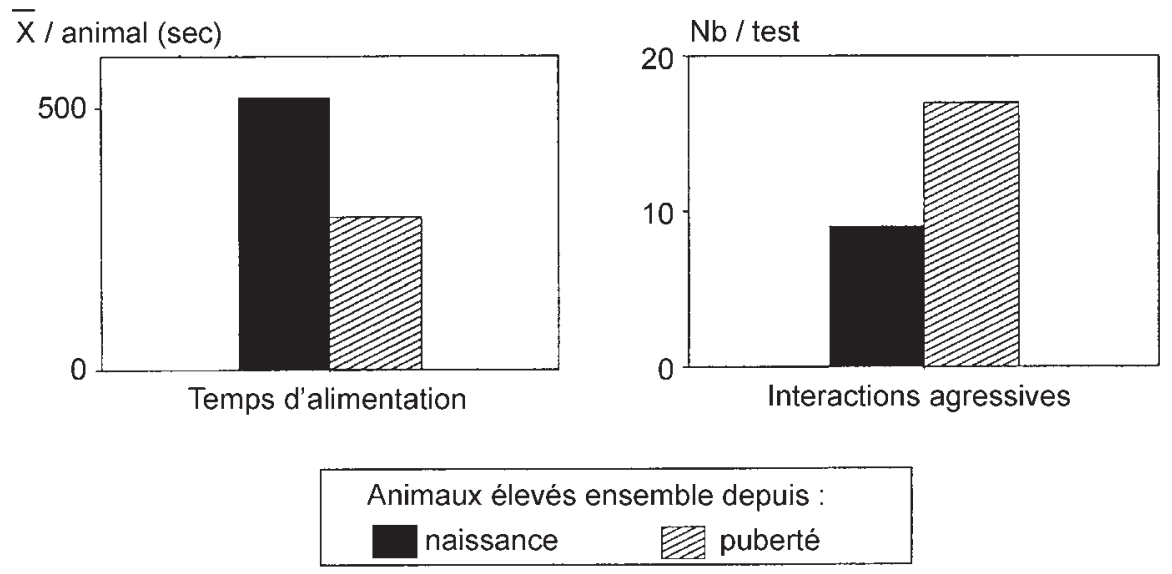

vité sociale et de la mémoire spatiale du leader. Peu de choses sont connues à propos du leadership spatial «volontaire» qui concerne les déplacements collectifs spontanés au pâturage. Ceci est probablement dû au fait que l'observation des déplacements spontanés survenant dans le groupe non perturbé exige une investigation coûteuse en terme de temps (Dumont et al sous presse). On a surtout observé le leadership spatial «forcé» qui concerne la relative permanence de l'ordre dans lequel des vaches laitières entrent dans la salle de traite, ou l'ordre de déplacement des d'animaux lorsqu'ils sont poussés par l'homme, dans le cas par exemple de changements de parcelles au pâturage. Dans ce cas, des ordres très répétables ont été mis en évidence, bien que les corrélations entre les différents ordres demeurent faibles (Albright et Arave 1997). De même, il n'y a qu'une faible corrélation entre leadership et rang hiérarchique. Lors des mouvements volontaires, ce sont en général les vaches de rang moyen qui sont en tête, les dominantes au centre et les subordonnées ferment la marche (Albright et Arave 1997). Durant les mouvements forcés, au contraire, ce sont plutôt les dominées (qui fuiraient l'homme) qui sont en tête.

\section{3 / Relations sociales et ges- tion de l'élevage : prise en compte de la dimension sociale pour concilier bien- être et production}

Les bovins sont élevés dans des conditions très variées de par le monde. Dans les pays en voie de développement, le bétail est souvent lié au pastoralisme. Dans les pays industrialisés, on trouve principalement deux modes de gestion. Dans les troupeaux allaitants, les vaches élèvent leur veau jusqu'aux environs de 4 à 9 mois. Il y a en général un mâle par troupeau. Lorsqu'ils ne sont pas utilisés pour la reproduction, les jeunes mâles (appelés broutards) sont réunis après le sevrage en unités d'engraissement qui se caractérisent entre autres par une forte densité sociale. Au contraire dans les races laitières, les veaux sont séparés de leur mère très tôt après la naissance (généralement avant l'âge de 3 jours) pour être placés en allaitement artificiel. Les veaux femelles sont élevés en groupe et rejoindront ultérieurement le groupe de vaches. Ces dernières sont en général regroupées selon leur niveau de pro- 
duction. Les veaux mâles sont utilisés pour la production de viande de veau (veau de boucherie), ou engraissés dans des ateliers spécialisés, comme les mâles issus de troupeaux allaitants.

Différents types génétiques de bovins sont utilisés pour ces différents modes de gestion, et semblent être adaptés à des environnements spécifiques que l'on peut qualifier de "niche écologique». Ainsi, ils diffèrent non seulement par leur capacité à produire de la viande ou du lait, mais aussi par leur comportement. Par exemple, les vaches de race Salers, utilisées pour la production de viande, ont un comportement maternel plus sélectif que les vaches de race Holstein (Le Neindre 1984). De même, les veaux Salers cherchent à maintenir un contact plus étroit avec leur mère que les veaux Holstein et ont plus de problèmes pour s'adapter à l’élevage artificiel.

Les bovins ont montré au cours de leur domestication, et surtout au cours des dernières décennies, des capacités d'adaptation importantes mais que l'on risque de dépasser à force d'artificialisation et d'intensification de leurs conditions de vie. Ces dernières sont à l'origine de tensions sociales qui peuvent avoir des répercussions au niveau de la productivité mais aussi du bienêtre des animaux. Les questions de bien-être se posent effectivement plus en systèmes intensifs : c'est là que les attentes sociétales semblent les plus fortes et ce sont ces productions qui sont visées lors de l'élaboration des réglementations. Toutefois, les systèmes extensifs ne sont pas sans problème concernant le bien-être des ani-

Figure 10. Effet de différents types de séparations sur le temps d'alimentation chez les bovins en fonction de leur niveau de dominance (Bouissou M.-F., 1970).

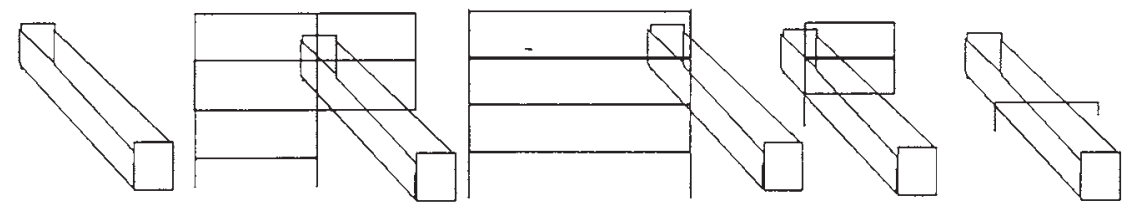

Temps d'alimentation (min)

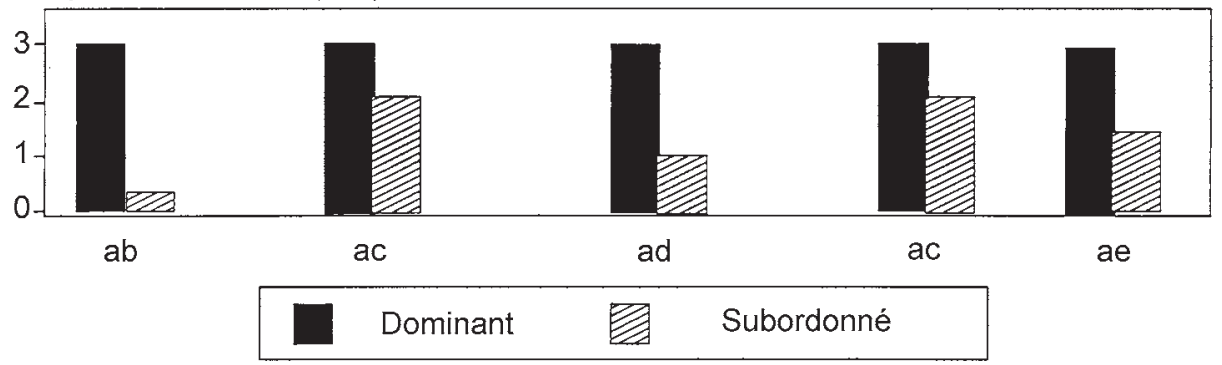

La protection au niveau de la tête est importante ; elle peut même être réduite à un niveau symbolique : une simple barre au dessus de l'auge.

maux. Or, ils sont voués à se développer puisqu'ils répondent aux orientations européennes en matière de gestion de l'environnement. Après avoir décrit les cas d'inconfort dont les relations sociales seraient à l'origine en élevage intensif, nous discuterons des moyens d'action possibles pour réduire ces tensions, en particulier grâce au respect des caractéristiques sociales des bovins. En outre, nous verrons en quoi le groupe social peut être considéré, notamment en élevage extensif, comme l'interface entre l'environnement physique et l'individu, permettant à ce dernier une meilleure adaptation.

\section{1 / Tensions sociales}

Une des caractéristiques de l'élevage intensif est l'entretien des animaux à de fortes densités, ce qui réduit les coûts de construction et de main-d'oeuvre. Dans de telles conditions, les animaux sont incapables de maintenir leurs «distances sociales», et ont du mal à éviter les plus dominants, ce qui provoque entre autres un accroissement des conduites agressives. Lorsque les conditions deviennent trop contraignantes, les phénomènes de dominan$c e$, qui ont normalement une fonction adaptative, ne peuvent plus jouer leur rôle. Ils peuvent même avoir de nombreuses conséquences défavorables pour les animaux subordonnés chez lesquels un accroissement des réponses physiologiques indicatrices de stress est observé (Hafez et Bouissou 1975, Kondo et al 1989). Dans les cas extrêmes, les animaux dominés peuvent subir un véritable stress social qui se traduit en particulier par une augmentation de l'activité des glandes surrénales

bolique : une simple barre au dessus de l'auge. dont la zone corticale s'hypertrophie. On a également trouvé une corrélation inverse entre le taux de leucocytes dans le lait (qui est un indicateur de stress) et le rang social des vaches (Kay et al 1977).

Enfin, les bovins entretenus en conditions intensives peuvent développer des comportements qualifiés d'anormaux comme la succion mutuelle par exemple. Cette succion mutuelle des oreilles, du prépuce ou du scrotum survient souvent après la distribution de lait chez les veaux élevés au seau. La succion du prépuce peut conduire à l'absorption d'urine et à des abcès. L'alimentation par des tétines artificielles réduit ces tétées non nutritives. Lorsqu'il se produit chez l'adulte, ce phénomène peut être la cause de problèmes sanitaires pour la mamelle de l'animal cible. L'isolement social accroît les comportements oraux non nutritifs comme le léchage d'objets disponibles dans l'environnement, et le «jeu de langue» (tongue-rolling). Par ailleurs, dans les groupes unisexués de mâles, on observe parfois un comportement excessif de monte connu sous le nom de «bullersteer syndrome». Un mâle particulier devient la cible des chevauchements incessants de ses compagnons, il peut alors être gravement blessé et même en mourir si l'on n'intervient pas assez tôt.

C'est sans doute dans le domaine de l'alimentation que les effets de la hiérarchie se font le plus sentir et ont été les plus étudiés. De nombreux auteurs ont montré l'avantage dont jouissaient les animaux dominants, lorsque l'alimentation est rationnée et lors d'une supplémentation. Si la compétition est forte, il y a une corrélation positive entre le temps d'alimentation et le rang social. La réduction du temps d'alimentation généralement manifestée par les animaux dominés a des conséquences sur leur croissance et leur productivité. Les animaux dominants, non seulement mangent pendant plus longtemps, mais sont également moins dérangés. Or, le fait de placer des séparations, même de dimensions limitées au niveau de la tête, réduit l'effet négatif de la dominance, et permet au dominé (qui se sent protégé) d'augmenter son temps d'alimentation (figure 10).

Les relations de dominance ont également un effet négatif sur l'activité sexuelle des taureaux. Si le nombre de vaches en chaleurs est important les dominés parviennent à s'accoupler. Par contre dès que ce nombre est faible (3 femelles ou moins), le mâle domi- 
nant interrompt les $9 / 10^{\mathrm{e}}$ des tentatives d'accouplement de ses subordonnés. On voit immédiatement les conséquences négatives que cela peut avoir si la fertilité du dominant est faible. Des études sur des troupeaux comportant plusieurs mâles ont montré qu'une minorité $(1 / 3)$ des taureaux engendrait de 65 à $100 \%$ des descendants (de Blockey 1978).

Les phénomènes de dominance interviennent également dans l'utilisation de l'espace. Même dans les conditions de l'élevage en stabulation, les bovins montrent des préférences très nettes pour certaines zones d'alimentation ou de repos. Lorsque l'aire de repos est divisée en logettes, les vaches dominantes jouissent de priorité pour le choix de l'emplacement, et les dominées sont souvent reléguées dans les zones les moins favorables : zones de passage où elles seront dérangées, courants d'air... Le simple fait qu'un animal dominant stationne devant une porte empêche les dominés d'utiliser les aires de repos, abritées ou ombragées, et provoque une réduction de leur temps d'alimentation au distributeur de nourriture.

En conclusion, la qualité de vie des animaux dominés peut donc être profondément affectée par leur statut social inférieur, en particulier dans les conditions d'élevage intensif. Ainsi la dominance sociale, qui devrait être adaptative et faciliter la vie en groupe, peut devenir source de problèmes graves lorsque les conditions d'élevage ne répondent plus aux exigences sociales des animaux.

Certaines pratiques de l'élevage intensif empêchent l'instauration d'une hiérarchie de dominance stable et durable. Ainsi, le regroupement d'animaux non familiers est une pratique courante en élevage. Or, le nombre des interactions agonistiques peut être décuplé dans les heures qui suivent. De plus, de nombreuses études ont montré que des modifications répétées des groupes sociaux entraînent des modifications physiologiques et comportementales indicatrices de stress. Chez les veaux de boucherie, des regroupements successifs, qui entraînent la rupture des liens sociaux et maintiennent les groupes en état d'instabilité sociale, se traduisent par une diminution des capacités des veaux à faire face à des changements de milieu (Boissy et al 2001a, Veissier et al 2001). De même, il est commun de réalloter les vaches laitières pendant la période de lactation selon leur niveau de production : bien que l'on considère souvent que cela n'affecte pas la production laitière, des études ont en fait mis en évidence une réduction de la courbe de production qui peut atteindre $4 \%$ au cours des cinq premiers jours (Hasegawa et al 1997).

Dans le cas de groupes de taille extrêmement importante (par exemple les «feedlots» américains et australiens), les animaux peuvent avoir des difficultés à mémoriser l'identité de leurs compagnons, ce qui empêche l'instauration d'une hiérarchie de dominance et se traduit alors par un accroissement des agressions.

A l'opposé des conditions de fortes densités sociales, l'élevage en isolement social n'est pas sans poser de nombreux problèmes tant chez le jeune que chez l'adulte. Chez les veaux de boucherie, le fait d’être élevé en isolement partiel a des effets importants sur la réactivité et le comportement social ultérieur des animaux. Les veaux élevés en case individuelle sont plus facilement effrayés, et ce d'autant plus qu'ils n'ont pas de contact visuel avec leurs voisins (Veissier et al 1997). Cette hyperréactivité peut occasionner des réponses de stress plus intenses lors des transports. En ce qui concerne le comportement social, certaines études ont mis en évidence une relative inadaptation et un statut social inférieur des animaux élevés initialement dans l'isolement. A l'âge adulte, la séparation d’avec les congénères entraîne également un accroissement à la fois de la locomotion, des vocalisations de détresse, une augmentation de la fréquence cardiaque et des niveaux de cortisol plasmatique, indicateurs d'inconfort (Boissy et Le Neindre 1997). Les éleveurs sont ainsi particulièrement exposés à des accidents lorsqu'ils tentent de séparer un individu de son groupe. Il existe cependant des différences entre races : les vaches Holsteins en particulier semblent moins perturbées que celles de races rustiques comme les Salers par la séparation d'avec leur groupe et s'adaptent plus facilement à diverses pratiques, telles que les robots de traite ou les distributeurs automatiques de nourriture (Le Neindre 1984).

\section{2 / Influences bénéfiques des relations sociales}

A côté des conséquences défavorables que nous venons d'évoquer, certaines relations sociales sont au contraire à l'origine d'une moindre agressivité et de tolérance mutuelle, même en cas de compétition : ce sont les relations d'affinité qui assurent la cohésion du groupe social.

Le développement des relations d'affinité, grâce au maintien de la structure du groupe établie dans le jeune âge, permet aux jeunes bovins en croissance de supporter plus facilement les fortes densités auxquelles ils sont généralement soumis en atelier d'engraissement, en minimisant les tensions sociales (Mounier et al sous presse). Plus généralement, la constitution d'un «noyau social» dès le plus jeune âge et le maintien de celui-ci au cours des différentes étapes de la vie de l'animal devraient permettre d'améliorer la tolérance mutuelle et en conséquence de réduire la fréquence des interactions agressives entre animaux adultes. Cette mesure devrait permettre d'éviter les écarts de production, que ce soit chez les jeunes bovins en engraissement ou chez les vaches laitières. Ainsi, les mélanges entre animaux devraient être évités au maximum. Lorsqu'ils sont indispensables, ils devraient être réalisés, non pas en introduisant individuellement de nouveaux animaux, mais en regroupant plusieurs noyaux sociaux entre eux. Il y a donc bien intérêt, d'une part à maintenir stables les relations de dominance-subordination au sein des groupes pour que celles-ci jouent leur rôle régulateur dans les situations de conflits, et d'autre part à favoriser le développement des relations d'affinités pour atténuer les effets négatifs de ces relations (Boissy et al 2001b).

Par ailleurs, les relations qu'entretient l'individu avec ses partenaires influencent également ses réponses aux événements non sociaux. Par exemple, lorsqu'une génisse est exposée à une situation nouvelle, la simple présence d'un partenaire familier, ou même de sa propre image reflétée par un miroir, suffit à diminuer ses réactions de néophobie (Boissy et Le Neindre 1990). De même, la facilité avec laquelle une génisse accepte d’être manipulée par un homme dépend de la présence de ses partenaires familiers (Grignard et al 2001). En outre, l'animal peut au travers de ses relations sociales acquérir de nouveaux comportements et ce de manière plus efficace que par apprentissage individuel. Ainsi, les apprentissages par observation et par imitation sont à la base de la transmission sociale des préférences et des évitements alimentaires (Nicol 1995). Par exemple des vaches accepteront plus rapidement de consommer des plantes qu'elles rencontrent pour la première fois, si elles 
se trouvent en présence de congénères qui ont appris précédemment à les consommer (Ralphs et al 1994). La mère joue en général un rôle essentiel dans ces phénomènes de transmission : cela à été surtout montré expérimentalement chez les ovins, mais il est fort probable qu'il en soit de même chez les bovins.

Le développement de l'extensification, en vue de valoriser les espaces impropres aux cultures, sollicite, au même titre que les conditions intensives, les capacités d'adaptation des animaux. Les animaux conduits en plein air doivent en effet acquérir un important niveau d'autonomie du fait d'une réduction de l'assistance par l'homme et d'une moindre maîtrise de l'environnement. Le renforcement de la cohésion du troupeau consécutif au maintien des liens sociaux établis dans le jeune âge module la manière dont le troupeau occupe l'espace et exploite le pâturage et permet une meilleure efficacité d'approvisionnement alimentaire. Enfin, une meilleure connaissance des déterminants comportementaux du leadership durant les phases de pâturage pourrait aboutir à en proposer une gestion moins empirique qui per- mette de prédire les «bons leaders» susceptibles d'entraîner leurs congénères vers les zones à faire exploiter en priorité

\section{Conclusion}

En élevage extensif, les contraintes au niveau social sont relativement faibles : les veaux restent avec leurs mères et avec d'autres adultes pendant plusieurs mois. Ils ont ainsi l’opportunité de développer leur comportement social de manière naturelle. Par contre, les pratiques de l'élevage intensif impliquent pour l'animal d'importantes contraintes sociales. Les relations de dominance-subordination sont exacerbées et semblent jouer un rôle majeur dans l'organisation et le fonctionnement du groupe, alors que les relations d'affinité sont actuellement peu prises en compte. L'importance relative de ces deux types de relations s'inverse dans les conditions plus extensives. Les conditions environnementales modulent donc l'expression des relations sociales, et en fonction des conditions un type de relation ou l'autre prévaut.
Les relations sociales ne doivent pas cependant pas être considérées comme n’ayant que des conséquences négatives en élevage. En effet, dans les conditions de fortes densités, le respect des relations d'affinité entre les animaux permet de minimiser les problèmes liés aux tensions inhérentes à la promiscuité. En outre, l'effet apaisant de la présence d'animaux familiers, l'apprentissage social et le leadership peuvent constituer des outils précieux pour améliorer l'adaptation de l'animal aux modifications de son environnement non social. Si les capacités d'adaptation des bovins domestiques et leur tolérance vis-à-vis des contraintes sont remarquables, ceci n'implique pas qu'elles soient sans limites. Nous devons définir des conduites d'élevage telles que les animaux puissent s'adapter au mieux en maximisant leur santé et plus généralement leur bien-être. Une meilleure prise en compte des règles de fonctionnement des groupes de bovins pour une exploitation à bon escient des caractéristiques sociales des animaux est un moyen efficace et réaliste pour réduire la pression sociale, voire même pour faciliter l'adaptation de l'individu aux diverses contraintes du milieu.

\section{Références}

Albright J. L., Arave C. W., 1997. The Behaviour of Cattle. CAB International, Wallingford, UK, 306 pp.

Baldwin B.A., 1977. Ability of goats and calves to distinguish between conspecific urine samples using olfaction. Appl. Anim. Ethol., 3, 145-150.

Blockey de M.A., Lade A.D., 1974. Social dominance relationships among young bulls in a test of rate of gain after weaning. Aust. Vet. J., 59, 435-437.

Boissy A., Bouissou M.-F., 1994. Effects of androgen treatment on behavioral and physiological responses of heifers to fear-eliciting situations. Horm. Behav., 28, 66-83.

Boissy A., Le Neindre P., 1990. Social influences on the reactivity of heifers: implications for learning abilities in operant conditioning. Appl. Anim. Behav. Sci., 25, 149-165.

Boissy A., Le Neindre P., 1997. Behavioral, cardiac and cortisol responses to brief peer separation and reunion in cattle. Physiol. Behav., 61, 693-699.

Boissy A., Nowak R., Orgeur P., Veissier I., 2001b. Les liens sociaux chez les ruminants d'élevage : limites et moyens d'action pour favoriser l'intégration de l'animal dans son milieu. INRA Prod. Anim., 14, 79-90.

Boissy A., Terlouw C., Le Neindre P., 1998. Presence of cues from stressed conspecifics increases reactivity to aversive events in cattle: evidence for the existence of alarm substances in urine. Physiol. Behav., 63, 489-495.

Boissy A., Veissier I., Roussel S., 2001a. Emotional reactivity affected by chronic stress : an experimental approach in calves subjected to environmental instability. Anim. Welf., 10, S175-S185.

Bouissou, M.-F., 1970. Rôle du contact physique dans la manifestation des relations hiérarchiques chez les bovins. Conséquences pratiques. Ann. Zootechn., 19, 279-285.

Bouissou, M.-F., 1975. Etablissement des relations de dominance-soumission chez les bovins domestiques. III. Rôle de l'expérience sociale. Zeitschrift für Tierpsychologie, 38, 419-435.

Bouissou, M.-F., 1977. Etude du développement des relations de dominance-subordination chez les bovins, à l'aide d'épreuves de compétition alimentaire. Biol. Behav., 2, 213-221.

Bouissou, M.-F., 1978. Effects of injections of testosterone propionate on dominance relationships in a group of cows. Horm. Behav., 11, 388400.

Bouissou M.-F., 1985. Contribution à l'étude des relations interindividuelles chez les Bovins domestiques femelles (Bos taurus L.). Thèse de Doctorat d'Etat, Université Paris VI, France, 366 pp.

Bouissou M.-F., Andrieu S., 1977. Etablissement des relations de dominance-soumission chez les bovins domestiques. IV.
Etablissement des relations chez les jeunes. Biol. Behav., 2, 97-107.

Bouissou M.-F., Andrieu S., 1978. Etablissement des relations préférentielles chez les bovins domestiques. Behaviour, 64, 148-157.

Bouissou M.-F., Gaudioso V., 1982. Effect of early androgen treatment on subsequent social behavior in heifers. Horm. Behav., 16, 132-146.

Bouissou M.-F., Hövels J., 1976a. Effet d'un contact précoce sur quelques aspects du comportement social des bovins domestiques. Biol. Behav., 1, 17-36.

Bouissou M.-F., Hövels J.H., 1976b. Effet des conditions d'élevage sur le comportement des génisses dans une situation de compétition alimentaire. Ann. Zootech., 25, 213-219.

Bouissou M.-F., Boissy A., Le Neindre P., Veissier I., 2001. The social behaviour of cattle. In: Social Behaviour in Farm Animals. Keeling L.J., Gonyou H.W., (Eds), CABI Publishing, CAB International, Wallingford, UK, 113145.

Cummins K.A., Myers L.J., 1991. Olfactory and visual cues, individual recognition, and social aggression in lactating dairy cows. J. Dairy Sci., 74, 301.

Daycard L., 1990. Structure sociale de la population de bovins sauvages de l'île 
d'Amsterdam, sud de l'Océan Indien. Rev. Ecol. (La Terre et la Vie), 45, 35-53.

Dumont B., Boissy A., Achard C., Sibbald A.M., Erhard H.W. (sous presse). Consistency of animal order in spontaneous group movements allows the measurement of leadership in a group of grazing heifers. Appl. Anim. Behav. Sci.

Entsu S., Dohi H., Yamada A., 1992. Visual acuity of cattle determined by the method of discrimination learning. Appl. Anim. Behav. Sci., 34, 1-10.

Grignard L., Boivin X., Boissy A., Le Neindre P., 2001. Do beef cattle react consistently to different handling situations? Appl. Anim. Behav. Sci., 71, 263-276.

Hafez E.S.E., Bouissou M.-F., 1975. The behaviour of cattle. In: Hafez E.S.E. (Ed) The Behaviour of Domestic Animals. Bailliere Tindall, London, 203-245.

Hall S.J.G., 1986. Chillingham cattle: dominance and affinities and access to supplementary food. Ethology, 71, 201-215.

Hall S.J.G., Moore G.F., 1986. Feral cattle of Swona, Orkney islands. Mammal Rev., 16, 8996.

Hasegawa N., Nishiwaki A., Sugawara K., Ito I., 1997. The effects of social exchange between two groups of lactating primiparous heifers on milk production, dominance order, behavior and adrenocortical response. Appl. Anim. Behav. Sci., 51, 15-27.

Kabuga, J.D., 1992. Social interactions in N'dama cows during periods of idling and supplementary feeding post-grazing. Appl. Anim. Behav. Sci., 34, 11-22.

Kay S.J., Collis K.A., Anderson J.C., Grant, A.J., 1977. The effect of intergroup movement of dairy cows on bulk-milk somatic cell numbers. J. Dairy Res., 44, 589-593.
Kiley M., 1972. The vocalisations of ungulates, their causation and function. Zeitschrift Tierpsychol., 31, 171-222.

Kondo S., Sekine J., Okubo M., Asahida Y., 1989. The effect of group size and space allowance on the agonistic and spacing behavior of cattle. Appl. Anim. Behav. Sci., 24, 127135 .

Lazo A., 1994. Social segregation and the maintenance of social stability in a feral cattle population. Anim. Behav., 48, 1133-1141.

Le Neindre P., 1984. La relation mère-jeune chez les bovins : influence de l'environnement social et de la race. Thèse de Doctorat d'Etat, Université de Rennes, France, 274 pp.

Le Neindre P., Sourd C., 1984. Influence of rearing conditions on subsequent social behaviour of Friesian and Salers heifers from birth to six months of age. Appl. Anim. Behav. Sci., 12, 43-52.

Lott D.F., Minta S.C., 1983. Random individual association and social group instability in American bison (Bison bison). Zeit. Tierpsychol., 61, 153-172.

Lucifero M., Janella G.G., Secchiari P., 1977. Origini, evoluzione, miglioramento e prospettive delle razza bovina Maremmana. Edagriocole (ed), Bologna, Italie.

Mansard, C., Bouissou, M.-F., 1980. Effect of olfactory bulbs removal on the establish ment of the dominance-submission relation hips in domestic cattle. Biol. Behav., 5, 169-178.

Meese G.B., Ewbank R., 1973. Exploratory behaviour and leadership in domestic pig. British Vet. J., 129, 251-259.

Mounier L., Veissier I., Boissy A. (sous presse) The false benefit of mixing finishing bulls to form groups of homogeneous weights. J. Anim. Sci.
Nicol C.J., 1995. The social transmission of information and behaviour. Appl. Anim. Behav. Sci., 44, 79-98.

Plusquellec P., Bouissou M.-F., 2001. Behavioural characteristics of two dairy breeds of cows selected (Herens) or not (Brune des Alpes) for fighting and dominance ability. Appl. Anim. Behav. Sci., 72, 1-21.

Ralphs M.H., Graham D., James L.F., 1994 Social facilitation influences cattle to graze locoweed. J. Range Managem., 47, 123-126.

Reinhardt V., 1981. Cohesive relationships in a cattle herd (Bos indicus). Behaviour, 77, 121 151.

Riol J.A., Sanchez J.M., Eguren V.G., Gaudioso V.R., 1989. Colour perception in fighting cattle. Appl. Anim. Behav. Sci., 23, 199-206.

Schloeth R., 1958. Le cycle annuel et le comportement social du taureau de Camargue. Mammalia, 22, 121-139.

Schloeth R, 1961. Das Sozialleben des Camargue Rindes. Zeit. Tierpsychol., 18, 574 627.

Stricklin W.R., 1983. Matrilinear social dominance and spatial relationships among Angus and Hereford cows. J. Dairy Sci., 57, 1397-1405.

Syme G.J., Syme L.A., 1979. Social Structure in Farm Animals. Elsevier Science Publications. 200p.

Veissier I., Chazal P., Pradel P., Le Neindre P., 1997. Providing social contacts and objects for nibbling moderates reactivity and oral behaviors in veal calves. Appl. Anim. Ethol., 75, 356-365.

Veissier I., Boissy A., dePassillé A.M., Rushen J., van Reenen C.G., Roussel S., Andanson S., Pradel P., 2001. Calves' responses to repeated social regrouping and relocation. J. Anim. Sci., 79, 2580-2593.

\section{Résumé}

Les bovins appartiennent à des espèces sociales et vivent en groupes permanents au sein desquels ils développent des relations stables. Ce haut niveau de socialisation, qui a probablement facilité leur domestication, permet à l'éleveur de gérer les animaux en groupe, leur reproduction et l'élevage des jeunes.

Les relations de dominance et celles d'affinité constituent la base des relations sociales chez les bovins domestiques. Elles se traduisent par des actes et des postures particulières. Les relations de dominance organisent les interactions agonistiques alors que les relations d'affinité s'expriment au travers d'interactions positives comme le toilettage mutuel. En conditions normales de groupe permanent les relations de dominance sont particulièrement stables et participent à la résolution à moindre coût des conflits. Cependant, leur expression peut être exacerbée par certaines conduites d'élevage entraînant alors des conséquences néfastes pour les dominés. Les relations d'affinité, qui se développent préférentiellement dans le jeune âge, assurent la cohésion ultérieure des groupes et permettent d'atténuer les éventuelles tensions.

Les importantes contraintes sociales imposées par les conditions modernes d'élevage peuvent être génératrices d'inconfort, voire de stress, qui altère les performances et le bien-être des bovins. Une meilleure connaissance des relations sociales et de leurs mécanismes constitue un outil précieux, pour améliorer l'intégration de l'animal à son groupe d'élevage, en assurant la stabilité des relations de dominance et en privilégiant les relations d'affinité. Par ailleurs, une meilleure gestion des relations au sein du groupe devrait permettre d'accroître l'adaptation des bovins aux conditions d'élevage grâce en particulier aux phénomènes d'entraînement (imitation, leadership) et d'apaisement social. 


\begin{abstract}
The social behaviour of cattle and its consequences on breeding

Cattle are social animals that live in large, highly organised groups with stable social relationships. This high level of socialisation that has probably been at the basis of their domestication helps the farmer to manage the herd, their reproduction and their growth.

The social organisation of cattle is characterised by dominance-subordination relationships and by preferential relationships. These two types of social relationships are expressed by specific behaviours and postures: dominance relationships are expressed throughout agonistic (offensive and defensive) interactions whereas preferential relationships are expressed throughout positive interactions such as mutual grooming. Under normal conditions of a permanent group, the dominance relationships are stable and help to resolve conflicts between animals caused by proximity. The preferential relationships essentially developed between calves during ontogeny, are responsible for the cohesion of the group and help to attenuate social tensions.

Modern husbandry practices impose constraints to the environment of cattle including disturbances of their social environment, which can induce stress and reduce their production and their welfare. The expression of the dominance relationships can be exacerbated by farming conditions that lead to negative influences on the subordinates. A better knowledge of social relationships can generate specific tools to alleviate problems due to social tension by insuring stability among the dominance relationships and by increasing preferential relationships. In addition, a better management of the relationships among the group should also provide useful means for increasing the adaptation of animals to their non-social environment through buffering effects (i.e., stress-reducing effect of peers) and social facilitations (e.g., leadership and social learning). A better respect of the social needs and the social abilities of cattle, ultimately, will help to ensure not only optimal production but also to maximise animal welfare.
\end{abstract}

BOUISSOU M.-F., BOISSY A. 2005. Le comportement social des bovins et ses conséquences en élevage. INRA Prod. Anim., 18, 87-99. 
